\title{
In the era of recombinant BMP, does additional anterior stabilization add value to a posterolateral fusion?
}

Authors Priyesh Dhoke1, Ben Goss², Satyen Mehta ${ }^{1}$, Sanela Stanojevic ${ }^{2}$, Richard Williams ${ }^{1,2}$

Institutions ${ }^{1}$ AOSpine Reference Centre, Princess Alexandra Hospital, Brisbane, Australia

${ }^{2}$ Queensland University of Technology, Brisbane, Australia

\begin{tabular}{l|l|}
\hline Final class of \\
evidence-treatment \\
Study design \\
\hline RCT \\
\hline Cohort \\
Case control \\
\hline Case series \\
\hline Methods \\
\hline Concealed allocation (RCT) \\
\hline Intention to treat (RCT) \\
\hline Blinded/independent \\
evaluation of primary outcome \\
\hline F/U $\geq 85 \%$ \\
\hline Adequate sample size & \\
\hline Control for confounding & \\
\hline Overall class of evidence & III \\
\hline
\end{tabular}

The definiton of the different classes of evidence is available on page 67.

\section{ABSTRACT}

Study design: Retrospective cohort study.

Clinical question or objective: Is there a benefit to additional transforaminal lumbar interbody fusion (TLIF) if a solid posterolateral (PL) fusion can be achieved with routine bone morphogenetic protein (BMP) use in low-grade spondylolisthesis?

Methods: We performed a retrospective review of patients who had undergone surgery for grade I or II lumbar spondylolisthesis stratified into two groups. Group 1 had 46 patients who underwent TLIF along with PL instrumented fusion. Group 2 had 40 patients who underwent PL instrumented fusion alone. In both groups, adequate posterior decompression with pedicle screw instrumentation was performed and rhBMP-7 was used. All patients were evaluated clinically using the Oswestry Disability Index (ODI) and by independent radiological examination at 3 and 12 months.

Results: At a minimum follow-up of 12 months, there was no statistically significant difference in the rate of fusion. In addition, there were no differences in the proportion of patients who had a minimal clinically significant difference in their ODI. There was a similar rate of complications between each cohort.

Conclusions: The use of BMP was associated with a high rate of PL lumbar fusion. In the presence of a PL fusion, there appears to be little clinical benefit to additional anterior TLIF in degenerative spondylolisthesis. 


\section{STUDY RATIONALE AND CONTEXT}

Prior to the widespread use of bone morphogenetic proteins (BMPs), lumbar interbody fusion augmented by posterior fixation was reported to improve patient outcome through indirect decompression of the intervertebral foramen and through increased likelihood of fusion success. These days, BMPs provide greater certainty in PL fusion, raising the question: Is there clinical value in anterior column support in low-grade spondylolisthesis?

\section{CLINICAL QUESTION}

Is there a benefit to additional transforaminal lumbar interbody fusion (TLIF) if a solid PL fusion can be achieved with routine BMP-7 use in low-grade spondylolisthesis?

Fig 1 Patient population and interventions compared.

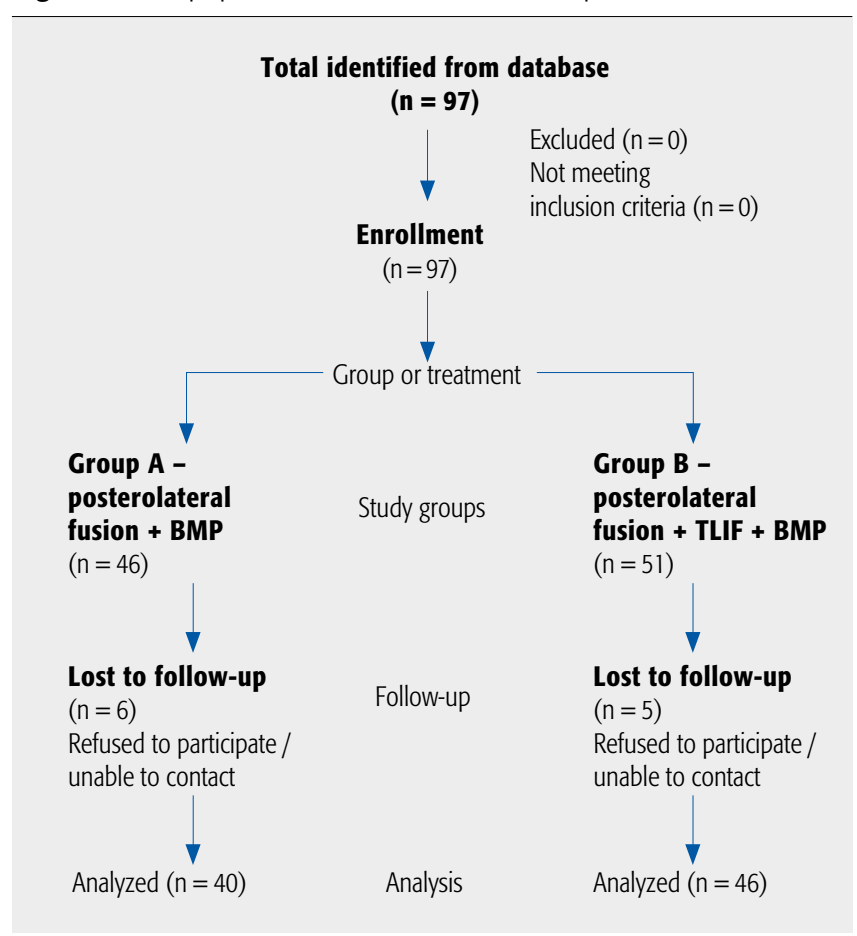

\section{METHODS}

Study design: A retrospective single-hospital, singlesurgeon cohort of patients treated for grade I or II spondylolisthesis by PL fusion with BMP-7 with or without TLIF and anterior BMP-7.

Inclusion criteria: Patients with grade I or II spondylolisthesis, PL grafting with BMP-7, aged between 30 and 90 years.

Exclusion criteria (Fig 1): high-grade spondylolisthesis (grade III or IV), isthmic spondylolisthesis, dysplastic spondylolisthesis, previous lumbar spine surgery, patient taking steroids, patient receiving chemotherapy, smokers (in the previous 6 months).

Patient population and interventions compared (Fig 1):

- The AOSpine Reference Centre based at Brisbane Private Hospital and The Princess Alexandra Hospital, Australia, maintains a prospective clinical database for every spinal operation undertaken.

- A review of patients operated between January 2006 and December 2009 for grades I and II degenerative spondylolisthesis identified 97 consecutive patients at Brisbane Private Hospital.

- All patients underwent posterior decompression and instrumentation with the Universal Spine System (Synthes, West Chester, PA, USA).

- From this cohort, 46 of 51 patients who underwent TLIF using Travios (Synthes, West Chester, PA, USA) with PL fusion with local autograft (one spinous process) and BMP-7 (one ampoule) (Stryker, Warsaw, IN, USA), placed both anteriorly inside the cage and PL, were available for follow-up.

- In a second group 40 of 46 patients who underwent PL fusion with local autograft and BMP-7 representing a change in preference by the treating surgeon were available for follow-up.

- The overall follow-up rate was $89 \%$.

- Assessment was performed unblinded to the modality of treatment.

- Complications were assessed as either major, requiring immediate additional intervention (eg, postoperative leg pain, adjacent segment symptoms requiring surgery, or postoperative infection requiring washout), or minor, requiring observation only (eg, dural tear or adjacent segment symptoms not requiring surgery). 


\section{Outcomes:}

- The Oswestry Disability Index (ODI) was recorded preoperatively, at 3 months of care, as is standard, and ODI was collected by telephone interview approximately 12 months postoperatively.

- Posterior fusion was assessed using the Lenke classification [1].

- Anterior fusion was assessed using the modified criteria of Lee et al [2].

- Intraoperative and postoperative complications were recorded in both groups.

\section{Analysis:}

- Fisher exact test was used to compare groups on the following outcomes: (1) the proportion of patients who improved a minimum clinically significant difference of $12.8 \%$ ODI at the various time points; (2), proportion of patients with successful fusion; and (3) proportion of patients with complications [3].

Additional methodological and technical details are provided in the Web Appendix at www.aospineorg/ebsj.

Table 1 Demographic and baseline characteristics of intervention groups.*

\begin{tabular}{llll}
\hline & TLIF + PL & PL alone & $\boldsymbol{P}$ \\
\hline $\mathrm{N}$ & 46 & 40 & \\
\hline Age, mean \pm SD, $y$ & $60 \pm 11$ & $66 \pm 10$ & .01 \\
\hline Female, No. (\%) & $18(39)$ & $21(52.5)$ & .17 \\
\hline Smoker, No. (\%) & $0(0)$ & $0(0)$ & 1.0 \\
\hline Levels fused & & & \\
\hline 1 & 32 & 18 & - \\
\hline 2 & 13 & 16 & - \\
\hline 3 & 1 & 6 & - \\
\hline
\end{tabular}

${ }^{*}$ TLIF (transforaminal lumbar interbody fusion) + PL (posterolateral fusion).

\section{RESULTS}

- Figure 1 indicates the number of patients in each group. Table 1 shows patient demographics; 40 patients had PL fusion with BMP-7, and 46 patients had TLIF and PL fusion with BMP-7.

- At 3 months, $23(57 \%)$ of 40 patients in the PL fusion group and $30(65 \%)$ of 46 patients in the TLIF+PL fusion group obtained a clinically significant benefit from the operation based on ODI (Table 2).

- After 12 months 21 (52\%) of 40 patients in the PL fusion group and $31(67 \%)$ of 46 in the TLIF+PL fusion group obtained a clinically significant benefit from the operation based on ODI (Table 2).

- Three $(7.5 \%)$ of 40 patients in the PL group were not fused posteriorly by Lenke criteria at 12 months.

- One $(2.2 \%)$ of 46 patients in the TLIF group was not fused posteriorly by Lenke criteria at 12 months.

- One $(2.2 \%)$ of 40 patients in the PL group were not fused anteriorly by Lee modified criteria at 12 months.

- Table 3 displays the rate of complications. A detailed breakdown of complications is included in the Web Appendix at www.aospine.org/ebsj.

- The results of our comparative study of the above two techniques showed that there was no difference in clinical outcome measured by ODI $(P=.188)$, in fusion rate $(P=.339)$ and complication rates. This supports the original hypothesis.

Table 2 ODI improvement and fusion status at follow-up.*

\begin{tabular}{llcll}
\hline & & TLIF + PL & \multicolumn{2}{c}{ PL alone } \\
\hline & $3 \mathrm{mo}$ & $12 \mathrm{mo}$ & $3 \mathrm{mo}$ & $12 \mathrm{mo}$ \\
\hline Patients improving MCID & $30 / 46$ & $31 / 46$ & $23 / 40$ & $21 / 40$ \\
ODI & $(65 \%)$ & $(67 \%)$ & $(57 \%)$ & $(52 \%)$ \\
(MCID=12.8) & & & & \\
\hline Fusion status & - & $45 / 46$ & - & $37 / 40$ \\
& & $(97.8 \%)$ & & $(92.5 \%)$ \\
\hline
\end{tabular}

* TLIF indicates transforaminal lumbar interbody fusion; PL. posterolateral; $M C I D$, minimal clinically significant difference; and ODI, Oswestry Disability Index.

Table 3 All complications.*

\begin{tabular}{llllll}
\hline & $\begin{array}{l}\text { Major } \\
\text { complications }\end{array}$ & $\begin{array}{l}\text { Minor } \\
\text { complications }\end{array}$ & $\begin{array}{l}\text { Early } \\
\text { complications }\end{array}$ & $\begin{array}{l}\text { Delayed } \\
\text { complications }\end{array}$ & $\begin{array}{l}\text { Complications requiring } \\
\text { surgical intervention }\end{array}$ \\
\hline PL & $8 / 40(20 \%)$ & $10 / 40(25 \%)$ & $8 / 40(20 \%)$ & $10 / 40(25 \%)$ & $6 / 40(15 \%)$ \\
\hline TLIF + PL & $7 / 46(15 \%)$ & $2 / 46(4 \%)$ & $4 / 46(7 \%)$ & $5 / 46(11 \%)$ & $7 / 56(15 \%)$ \\
\hline
\end{tabular}

${ }^{*} \mathrm{PL}$ indicates posterolateral; TLIF (transforaminal lumbar interbody fusion) + PL. 


\section{DISCUSSION}

- Of various surgical techniques for spondylolisthesis, TLIF is gaining popularity; although technically more challenging, as it involves circumferential fusion and restores disc height.

- Experimentally, $360^{\circ}$ fusion has been shown to provide significant biomechanical stability over isolated posterior fusion [4]. In our study, there was no significant difference in fusion rates, which may be the reason for the similar functional outcomes.

- Most studies in the past comparing PLF with PLIF for spondylolisthesis have found better functional results with PLIF [57]. This has been attributed to the additional anterior column support leading to higher-fusion rates provided by interbody fusion.

- Recombinant human BMP-7 is known to augment fusion rates in many clinical trials worldwide, although recently the safety of other BMPs, especially in anterior fusion, has been questioned [8]. This made us question the necessity of a more cumbersome and technically demanding and potentially unsafe TLIF/BMP-7 procedure over solid PL fusion with BMP-7.

- We showed that if a solid fusion can be achieved posteriorly with adjunct therapies, such as BMP-7, there is no significant difference in the results between the PLIF/PLF and the PLF-alone groups and it would seem reasonable to avoid the extra procedure and perform PLF alone.

- Strengths: Single hospital, single surgeon, similar techniques, and good follow-up.

- Limitations: The study was retrospective in design, computed tomography (CT) was not used to assess fusion status, and no clinical outcome measures were recorded in early postoperative period (ie, 1 day and lweek after surgery).

- Clinical relevance and impact: This study suggests that there may be some value in rethinking the role of primary circumferential fusion. There is a difference in the complication profile of BMP-7 and BMP-2 and as such a limitation of this study is that these results may not directly relate to the utility of BMP-2 in this application.

\section{SUMMARY AND CONCLUSIONS}

- In the presence of a solid posterior fusion, the additional support provided by an anterior fusion has no significant benefit in ODI in the mid-term to long-term.

- While there was no significant difference in complications, the study was not powered to detect this. What is important to note is that the range and severity of complications in each group are similar. 


\section{REFERENCES}

1. Lenke LG, Bridwell KH, Bullis D, et al (1992) Results of in situ fusion for isthmic spondylolisthesis. J Spinal Disord; 5(4):433-442.

2. Lee CK, Vessa P, Lee JK (1995) Chronic disabling low back pain syndrome caused by internal disc derangements. The results of disc excision and posterior lumbar interbody fusion. Spine; 20(3):356-361.

3. Copay AG, Glassman SD, Subach BR, et al (2008) Minimum clinically important difference in lumbar spine surgery patients: a choice of methods using the Oswestry Disability Index, Medical Outcomes Study questionnaire Short Form 36, and pain scales. Spine J; 8(6):968-974.

4. Crawford NR, Cagli S, Sonntag VK, et al (2001) Biomechanics of grade I degenerative lumbar spondylolisthesis. Part 1: in vitro model. J Neurosurg; 94 Suppl 1:45-50.

5. Dantas FL, Prandini MN, Ferreira MA (2007) Comparison between posterior lumbar fusion with pedicle screws and posterior lumbar interbody fusion with pedicle screws in adult spondylolisthesis. Arq Neuropsiquiatr; 65(3B): 764-770.

6. Suk SI, Lee CK, Kim WJ, et al (1997) Adding posterior lumbar interbody fusion to pedicle screw fixation and posterolateral fusion after decompression in spondylolytic spondylolisthesis. Spine; 22(2):210-219; discussion 219-220.

7. La Rosa G, Conti A, Cacciola F, et al (2003) Pedicle screw fixation for isthmic spondylolisthesis: does posterior lumbar interbody fusion improve outcome over posterolateral fusion? J Neurosurg; 99 Suppl 2:143-150.

8. Carragee EJ, Hurwitz EL, Weiner BK (2011) A critical review of recombinant human bone morphogenetic protein-2 trials in spinal surgery: emerging safety concerns and lessons learned. Spine J; 11(6):471-491.

\section{EDITORIAL PERSPECTIVE}

This is a retrospective review of patients with degenerative spondylolisthesis with stenosis who underwent a transforaminal lumbar interbody fusion (TLIF) with posterolateral fusion $(P L F)+$ bone morphogenetic protein $(B M P)-7$ or with a PLF + BMP-7.

The reviewers found this to be a relatively well-reported study.

One main concern with this study is the product, BMP-7, which was used. The Food and Drug Administration has not approved it for use in the United States, so for the US readership, this article will not be of practical value. The reviewers wanted to make sure that the results would not be misinterpreted and false extrapolations would be made to BMP-2. This would be erroneous. Bone morphogenetic protein-2 and -7 are different products and results from this study should not be used to draw conclusions about the utility of BMP-2.

A second concern raised was the notion that fusion rates could be attributed to BMP-7. There are many studies that have reported fusion rates without BMP-7, using just local bone that is similar to the present study. How are we to know that the reported fusion rates were attributable to BMP-7 and not the local bone? This would need clarification, and this may be difficult to do in absence of a non-BMP-7 control.

Goss and colleagues deserve kudos for their use of Oswestry Disability Index and the calculation of minimally important clinical differences for treatment. This sort of reporting is increasingly becoming the standard. 